\title{
Diez herramientas digitales para facilitar la evaluación formativa
}

\author{
María Eugenia Walss Aurioles \\ Directora académica del Departamento de Ciencias de preparatoria del \\ Instituto Tecnológico y de Estudios Superiores de Monterrey Campus Laguna
}

walss@tec.mx

\section{Extracto}

Este artículo sugiere 10 herramientas digitales que permiten al profesor explotar su creatividad en el diseño de actividades de evaluación formativa y compartida. Para que una evaluación cumpla su función formativa debe proveer al alumno información que le permita tomar decisiones para realizar ajustes en su proceso de aprendizaje. La tecnología habilita la integración de la evaluación al proceso de enseñanza facilitando la retroalimentación, automatizando actividades que de otra manera consumen mucho tiempo del docente. Las herramientas seleccionadas permiten generar una gran variedad de tareas que favorecen la autoevaluación, la cooperación y la retroalimentación. Se recomiendan tecnologías gratuitas o de bajo costo, con posibilidad de integración a plataformas digitales didácticas, adaptables en todos los niveles educativos.

Palabras clave: evaluación formativa; retroalimentación; educación; herramientas tecnológicas; analítica del aprendizaje. 


\title{
Ten digital tools to promote formative assessment
}

\author{
María Eugenia Walss Aurioles
}

\begin{abstract}
This article suggests 10 digital tools that encourage teachers to exploit their creativity to design formative assessment activities. For an assessment to fulfill a formative function it must provide the students with information that empower them to make decisions and adjustments in their learning process. Technology enables embedding evaluation during instruction and simplifies feedback, automatizing activities that otherwise are time-consuming for teachers. The selected tools allow the development of a great variety of assignments that promote self-evaluation, cooperation and feedback. The review recommends free or low-cost technology, that support learning management system integration and adaptable to all learning levels.
\end{abstract}

Keywords: formative evaluation; feedback; education; digital tools; learning analytics.

Citation: Walss Aurioles, M. ${ }^{a}$ E. (2021). Ten digital tools to facilitate formative assessment. Tecnología, Ciencia y Educación, 18, 127-139. 


\section{Sumario}

1. La evaluación formativa y compartida

2. Tecnologías digitales que promueven la evaluación formativa y compartida

2.1. EDpuzzle

2.2. Flipgrid

2.3. Genially

2.4. Mentimeter

2.5. Nearpod

2.6. Quizlet

2.7. Piazza

2.8. Socrative

2.9. WebAssign

2.10. Wheeldecide

3. Conclusión

Referencias bibliográficas 


\section{La evaluación formativa y compartida}

La evaluación educativa se rige por una lógica: queremos decidir si estamos logrando lo que nos proponemos con nuestro curso, necesitamos hacer una afirmación valorativa sobre la situación, tomamos en consideración ciertas dimensiones, recogemos evidencias y las analizamos para establecer una conclusión (Ravela et al., 2017). Hace más de medio siglo que Scriven (1967) atribuyó a la evaluación dos finalidades, una a la que llamó "sumativa» y otra que denominó «formativa», concediéndoles igual importancia a ambos roles, ya que se obtiene retroalimentación de cada uno de ellos. Aunque reconocía la importancia de los dos objetivos, recomendaba que los evaluadores formativos no se involucraran en la evaluación sumativa e incluso sostenía que los instructores no podían ser buenos jueces de su propia práctica. Wiliam (2000) explica que Bloom, Hastings y Madaus, en 1971, fueron los primeros en definir la «evaluación sumativa» como cuestionarios administrados al final de las unidades o los cursos con la intención de calificar, certificar o incluso evaluar la efectividad del currículo, contrastándola con la «evaluación formativa», cuya finalidad es la mejora continua. Aunque esta distinción no parece excepcional, provocó una dicotomía que sostenía que se requerían dos sistemas de evaluación separados e incluso incompatibles. Wiliam sugiere tres rutas para mitigar esta tensión:

- Incrementar la evidencia para que un mayor número de resultados puedan ser evaluados.

- Dar a conocer a los estudiantes que están siendo evaluados.

- Cambiar el foco de atención del control de calidad al aseguramiento de la calidad, basado en la capacidad de autoevaluación del alumno.

La autoevaluación, lejos de ser un anexo de la evaluación formativa eficiente, constituye el núcleo del proceso. La condición esencial para que una evaluación tenga una función formativa es su capacidad de proveer evidencia en relación con lo que el alumno debe hacer a continuación para cerrar la brecha en su desempeño de manera efectiva. En este sentido, la evaluación formativa es prospectiva; contiene en sí misma una guía para tomar acción dirigida hacia la reevaluación (Wiliam, 2000). Ravela et al. (2017) tradujeron el término «reevaluación» al español como
Para que una retroalimentación sea efectiva debe responder a tres preguntas de los aprendices: ¿cuáles son los objetivos?, ¿cómo me estoy desempeñando? y ¿qué debo hacer a continuación? Un ambiente de aprendizaje ideal es aquel en el que tanto los profesores como los alumnos buscan las respuestas a todas estas preguntas 
«devolución», lo cual resulta muy acertado para denotar la circularidad del proceso. Hattie y Timperley (2007) sugieren que para que una retroalimentación sea efectiva debe responder a tres preguntas de los aprendices: ¿cuáles son los objetivos?, ¿cómo me estoy desempeñando? y ¿qué debo hacer a continuación? Un ambiente de aprendizaje ideal es aquel en el que tanto los profesores como los alumnos buscan las respuestas a todas estas preguntas.

Díaz y Hernández (2002) indican que la función central del docente consiste en orientar y guiar la actividad mental constructiva de sus alumnos, a quienes proporcionará una ayuda pedagógica ajustada. El mecanismo central por medio del cual se propicia el aprendizaje es la «transferencia de responsabilidad», que se refiere al nivel de responsabilidad de los estudiantes para lograr sus metas o propósitos. Para que esta transferencia sea eficaz es necesario que los docentes pongan en práctica el concepto de «evaluación formativa y compartida» (EFC). GarcíaJiménez (2015) asevera que los estudiantes aprenden a revisar su propio trabajo y a enfocar por sí mismos cómo deben afrontar la tarea actual y las futuras si la información que se proporciona cumple una función facilitadora. Define «proalimentación» como el uso que le da el estudiante a la información que tiene sobre su trabajo para tomar decisiones de regulación que le permitan alcanzar el nivel de referencia fijado. La proalimentación favorece que el alumno ingrese en un ciclo recursivo o espiral que conduce de la retroalimentación a la autorregulación y a la metacognición.

Alcalá et al. (2019) enlistan cinco razones por las que es necesario aplicar procesos de EFC:

- Permite al alumno ser más consciente de lo que se aprende.

- Empodera al discente para autorregular su aprendizaje.

- Promueve diferentes capacidades y competencias en el aprendizaje.

- Ofrece diversidad de canales de retroalimentación.

- Mejora de la práctica docente.

\section{Tecnologías digitales que promueven la evaluación forma- tiva y compartida}

En la actualidad, la ubicuidad de recursos tecnológicos, como ordenadores, tablets, teléfonos móviles y acceso a internet, democratiza el uso de «herramientas digitales» que promuevan la EFC. El uso de tecnología permite la administración de gran cantidad de 
datos, lo que se conoce como «analítica del aprendizaje» (learning analytics), que proveen a los docentes con una mina de información para identificar en qué nivel de desarrollo se encuentra cada alumno con el fin de proporcionarle el andamiaje y la mediación personalizada que requiere. Lo ideal sería que la evaluación sumativa se aplicara hasta que el discente haya desarrollado la competencia requerida. Hattie y Timperley (2007) reconocen que dar y recibir retroalimentación requiere de muchas habilidades por parte de los docentes y los alumnos; asimismo, aseveran que las formas más efectivas de retroalimentación están relacionadas con el logro de objetivos y proveen indicaciones a los aprendices en forma de vídeo, audio o asistidos por ordenador. La tecnología ayuda a automatizar actividades que consumen mucho tiempo, de manera que los docentes pueden dedicar este tiempo a proveer oportunidades de aprendizaje enriquecedoras.

Cosi et al. (2020) aseveran que las herramientas digitales auxilian en tres factores que afectan a la calidad de la retroalimentación proporcionada a los estudiantes:

- Tiempo. La retroalimentación es inmediata.

- Formato. La devolución puede entregarse en formato de texto, audio o vídeo.

- Detalle. Las herramientas digitales permiten dar acceso a múltiples recursos adicionales que están disponibles en internet para proporcionar al alumno más información.

Cosi et al. (2020) aseveran que las herramientas digitales auxilian en tres factores que afectan a la calidad de la retroalimentación proporcionada a los estudiantes: tiempo, formato y detalle

Las «tareas de aprendizaje» son los elementos clave sobre los que se construye el proceso de evaluación formativa de los alumnos
Las «tareas de aprendizaje» son los elementos clave sobre los que se construye el proceso de evaluación formativa. Ravela et al. (2017) categorizan las actividades de evaluación en función de los procesos cognitivos que el alumno utiliza para resolverlos:

- Recordar y reproducir.

- Utilizar o aplicar.

- Construir significados y comprender.

- Valorar y evaluar.

- Diseñar o crear. 
Aseveran que el nivel cognitivo que alcanza el estudiante no está condicionado por el tipo de instrumento que se utiliza, es decir, un cuestionario de opción múltiple puede diseñarse para el nivel uno, pero también para el nivel cuatro. Lo importante es que los estudiantes deben verse involucrados en una diversidad de situaciones didácticas para que el aprendizaje resulte significativo.

\section{La tecnología nos permite crear una gran variedad de estrategias de aprendizaje que se adaptan a los diferentes procesos cognitivos y estilos de aprendizaje}

La tecnología nos permite crear una gran variedad de estrategias de aprendizaje que se adaptan a los diferentes procesos cognitivos y estilos de aprendizaje. En el diseño de herramientas que permitan incidir en el proceso de enseñanza-aprendizaje se debe considerar:

- El que establece la línea base, el cambio que hay que lograr, y que se lleva a cabo en un inicio.

- El que representa el proceso de construcción del contenido que hay que aprender.

- El que permite ejercitar el contenido construido para conservarlo en la memoria.

- El que evidencia que el contenido se aplica en otros contextos o situaciones.

A continuación, se presentan 10 herramientas tecnológicas que facilitan a los docentes crear actividades para establecer el objetivo de aprendizaje, construir y ejercitar el contenido y evidenciar la aplicación del conocimiento en diferentes contextos. La selección está basada en la variedad, en la facilidad de uso tanto para profesores como para alumnos, en la facilidad de acceso desde cualquier dispositivo y en la posibilidad de integración con plataformas digitales didácticas (learning management system [LMS]). Se ha dado preferencia a aquellas tecnologías que son gratuitas o de costo accesible, que facilitan que los estudiantes modifiquen sus tareas a partir de la retroalimentación recibida y que el profesor pueda ocultar o liberar información en función del análisis de datos de aprendizaje para cada alumno. Las tecnologías recomendadas se presentan en orden alfabético.

\author{
En este trabajo se presentan 10 \\ herramientas tecnológicas que \\ facilitan a los docentes crear \\ actividades para establecer el \\ objetivo de aprendizaje, construir \\ y ejercitar el contenido y evidenciar \\ la aplicación del conocimiento \\ en diferentes contextos
}

\subsection{EDpuzzle}

Es una herramienta en línea (https://edpuzzle.com) que permite a docentes y estudiantes colocar elementos interactivos en un vídeo preexistente de una gran variedad de fuentes, como TED, YouTube, KhanAcademy, National Geographic, o en vídeos propios. La tecnología 
permite recortar los vídeos de manera que se elimina lo que no resulta esencial para el aprendizaje. También es posible grabar audio con explicaciones propias que complementan o eliminan el audio original. La característica más interesante es que permite hacer cortes en el vídeo para introducir preguntas de opción múltiple, preguntas abiertas y notas. Los vídeos se comparten generando un código en una sesión en vivo o un enlace para que los alumnos accedan de manera asíncrona. Tiene integración con Google Classroom, Canvas, Blackboard, Schoology, Moodle, entre otras LMS. Genera reportes de progreso, calificación, tiempo empleado, entrega tardía por grupo y por alumno, descargables en formato CSV. En la versión gratuita se pueden almacenar 20 vídeos. Resulta muy útil para diseñar clases de aula invertida y aprendizaje personalizado al ritmo de cada estudiante.

\subsection{Flipgrid}

Es una plataforma de aprendizaje gratuita en línea (https://info.flipgrid.com/) que permite a los educadores hacer una pregunta a la que los alumnos contestan con un vídeo. Es una herramienta muy amigable que puede utilizarse en todos los niveles educativos, ya que permite crear discusiones en línea. Por seguridad de los alumnos menores de edad es necesario que los padres de familia den su consentimiento. Para cada grupo se crea un grid en el cual se introducen preguntas en forma de tópicos. El número de tópicos es ilimitado. Pueden presentarse en formato de texto e incluir recursos como imágenes, vídeos, emoticonos o archivos anexos. Cada tema acepta un número ilimitado de respuestas. Los estudiantes responden por medio de la aplicación Flipgrid o en línea con cualquier dispositivo que tenga cámara. También pueden subir vídeos pregrabados. El tiempo de las respuestas puede limitarse de 15 segundos a 5 minutos. El instructor puede otorgar permiso para que los alumnos también respondan a sus compañeros. El rol de copiloto permite que más de un docente pueda moderar el grid. La plataforma tiene tutoriales para ayudar a los docentes y estudiantes a familiarizarse con su uso. Como con cualquier herramienta de comunicación, es necesario crear una cultura de respeto estableciendo reglas y expectativas claras antes de comenzar.

\subsection{Genially}

Es una herramienta en línea (https://www.genial.ly/en) para crear contenido interactivo. El plan gratuito permite creaciones ilimitadas, aunque no tiene acceso a las plantillas premium que resultan muy atractivas. Esta herramienta contiene modelos prediseñados para presentaciones, reportes, cuestionarios, unidades didácticas, gamificación, imágenes interactivas, infografías, guías y presentaciones en vídeo. Los diseños son visualmente atractivos y muy sencillos de modificar. El plan gratuito no permite descargar el contenido creado, sin embargo, se puede compartir en línea por medio de un enlace. El contenido interactivo es una excelente estrategia de evaluación formativa, ya que capta la atención de los alumnos y los mantiene activos y concentrados más tiempo en la tarea. Además, estimula la creatividad 
del docente para dar retroalimentación al alumno de manera lúdica y visual. La plataforma ofrece formación gratuita para los profesores. Las estadísticas de monitoreo de datos analíticos solo están disponibles en plan máster.

\subsection{Mentimeter}

Es un recurso digital en línea (https://www.mentimeter.com/) para crear presentaciones interactivas en las que se incluyen cuestionarios de opción múltiple, preguntas abiertas, nubes de palabras, escalas Likert, ranking y selección de imágenes. Además, permite recibir preguntas de la audiencia y crear competencias con cuestionarios de opción múltiple y preguntas abiertas. La herramienta genera un código de acceso, un enlace y un código QR para que los participantes puedan ingresar en menti.com a la presentación y participar. La versión gratuita limita el número de preguntas por presentación, la importación de plantillas de PowerPoint, Keynote o Google Slides y la exportación de respuestas a Excel. Solo es posible exportarlas en PDF. Se recomienda para presentaciones en las que la cantidad de información requiere pausas activas para fomentar la participación de todos o para recopilar información sobre la comprensión de conceptos clave, permitiendo a los alumnos realizar preguntas en tiempo real sin interrumpir la presentación o exponerse ante sus compañeros.

\subsection{Nearpod}

Es una aplicación, disponible también en línea (https://nearpod.com/), para crear presentaciones multimedia interactivas. La aplicación es compatible con todas las plataformas. Posibilita descargar vídeos, imágenes en 2D y 3D, audioclips, archivos PDF; subir presentaciones en Sway, Keynote, PowerPoint y Google Slides. También habilita incrustar en las diapositivas páginas web, simuladores PhET, recorridos virtuales y Twitter. Además, permite crear actividades de Flipgrid, pizarras colaborativas, dibujar, memoria, llenar los espacios, preguntas abiertas y de opción múltiple. En la modalidad gratuita, el dispositivo del alumno es administrado por el profesor, quien tiene el control de la presentación. Genera reportes con las respuestas de los alumnos, descargables en formato PDF o Excel. Los alumnos pueden acceder mediante un código o con un enlace. La versión de paga ofrece la opción de que el alumno avance a su propio ritmo y tiene integración con Google Classroom, Canvas, Schoology, itsLearning y Blackboard. Es una aplicación muy versátil en la que se pueden integrar actividades generadas con otras herramientas.

\subsection{Quizlet}

Es una aplicación compatible con todas las plataformas para crear fichas educativas y unidades de estudio, también disponible en página web (https://quizlet.com/es). El profesor crea 
tarjetas con los conceptos importantes de una unidad. El programa genera fichas con cada término y los combina para generar cuestionarios de opción múltiple, respuesta abierta, reproducción en audio para verificar ortografía, pruebas con formato combinado, actividades de relacionar y juegos de competencia entre los estudiantes. Los alumnos revisan las unidades de estudio de manera individual a su propio ritmo, repasando los conceptos en los diferentes formatos que ofrece la plataforma para autoevaluar su aprendizaje. Las unidades se pueden compartir mediante enlaces, Google Classroom y Remind. También permite crear clases dentro de la misma aplicación. La versión gratuita no incluye los reportes de avance por alumno. Esta herramienta didáctica es útil para introducir conceptos de manera variada y lúdica.

\subsection{Piazza}

Es un sistema de gestión de aprendizaje en línea gratuito (https://piazza.com/) con formato Wiki, que favorece la comunicación y la colaboración en un mismo espacio. Es un foro para que los alumnos hagan preguntas y obtengan respuestas por parte de sus compañeros y de su profesor. Disminuye respuestas repetitivas por parte del docente y aumenta la responsabilidad compartida entre los estudiantes, quienes incrementan su comprensión de un tema al intentar explicarlo a sus pares. Los instructores tienen la facultad de aprobar las respuestas de los alumnos. También ofrece opciones para realizar encuestas, discusiones y enviar mensajes. La plataforma genera estadísticas de participación y tiempos de respuesta y permite la integración con todos los LMS. Esta herramienta se recomienda para alumnos mayores, ya que su uso puede resultar complicado para los pequeños, aunque ofrece muchos tutoriales para docentes y estudiantes.

\subsection{Socrative}

Esta aplicación gratuita para dispositivos móviles de Mastery Connect está también disponible en línea (www.socrative.com). La versión del docente permite construir exámenes de opción múltiple, preguntas abiertas, falso/verdadero y pase de salida. Los cuestionarios se crean fácilmente. El docente puede incluir una explicación para dar retroalimentación adicional. Los alumnos acceden a la aplicación mediante un código proporcionado por el docente. Los resultados se observan en tiempo real y la aplicación genera reportes que incluyen el porcentaje de éxito por estudiante y por pregunta, descargables en formato CSV. El pase de salida es una excelente herramienta para medir la comprensión de los temas vistos en clase. Consiste en tres preguntas (en inglés):

- ¿En qué grado comprendiste el material de hoy?

- Totalmente.

- Bastante bien. 
- No muy bien.

- No entendí nada.

- ¿Qué aprendiste en la clase de hoy? (Pregunta abierta).

- Por favor, contesta la pregunta del docente. (Pregunta abierta).

\subsection{WebAssign}

Esta plataforma de aprendizaje en línea de Cengage (https://www.webassign.net/) permite a los instructores diseñar asignaturas y exámenes que proveen a los alumnos con retroalimentación inmediata. Los alumnos acceden a la plataforma mediante un LMS o en línea con un código de acceso. El profesor escoge un libro de texto con enfoque STEM (ciencia, tecnología, ingeniería y matemáticas). El catálogo cuenta con más de 900 libros que contienen preguntas, ejercicios, simulaciones, tutoriales, etc. Muchos de ellos también cuentan con e-book. El costo de la plataforma para los alumnos representa una fracción de lo que cuesta el libro de texto físico. Es una herramienta muy flexible que permite al docente diseñar asignaturas en las que cada alumno tiene problemas con datos distintos, por lo que el aprendizaje es personalizado. Incluye ejercicios de pregunta abierta, con opción de subir el procedimiento si se requiere, y ejercicios en los que se solicitan las respuestas de pasos intermedios. El profesor decide cuántas oportunidades tiene el estudiante para cada ejercicio. También puede darles acceso a la respuesta y a la solución paso a paso. Facilita a los alumnos tutoriales, vídeos e incluso ayuda en línea, lo cual resulta una excelente manera de dar retroalimentación y preparar a los discentes para la evaluación sumativa. Se recomienda que tanto la evaluación formativa como la sumativa se realicen en la plataforma para que los alumnos se familiaricen con el uso de la tecnología. Cuenta con herramientas de análisis muy poderosas que permiten monitorear el avance de cada estudiante, el tiempo que tarda en responder, el número de intentos y las estadísticas por alumnos y por grupo. Los reportes se pueden descargar en formato CSV.

\subsection{Wheeldecide}

Es una página online gratuita (https://wheeldecide.com/) que permite tomar decisiones de manera aleatoria. Aunque no tiene fines académicos como las anteriores, es una manera entretenida de motivar la participación de los alumnos. Es posible subir la lista de alumnos de una clase (máximo 100) para decidir quién contesta una pregunta o utilizar ruedas creadas para juegos. Se recomienda para clases a distancia con el fin de evitar que los alumnos se distraigan o desconecten de la sesión, presentando a los discentes preguntas que permitan al docente monitorear la efectividad del diseño instruccional. 


\section{Conclusión}

Tanto la evaluación formativa como la sumativa forman parte de los procesos de enseñanza y aprendizaje. Ambas proporcionan retroalimentación valiosa para el alumno y pueden considerarse como parte de un continuo entrelazado. Se debe partir del principio de que la educación no empieza por el contenido que se quiere enseñar, sino por las relaciones que se establecen entre un educando y un educador, de ahí que en la evaluación lo más importante sea intervenir en el aprendizaje de manera constante. Para hacerlo, el docente parte de una toma de decisiones reflexiva y dinámica que incluye la observación de lo que el discente sabe hacer desde el inicio, en el proceso y en el resultado; identificando qué se hace, qué se alcanza y qué falta por obtener; detectando, a su vez, el acierto para continuarlo y el error para evitarlo. Ello se lleva a cabo haciendo uso de instrumentos y herramientas que resultan útiles para observar lo que los estudiantes pueden hacer bien, lo que hacen con errores, lo que realizan con ayuda y lo que no pueden llevar a cabo incluso con ayuda (Frade, 2011).

La evaluación apoyada en la tecnología es una competencia que los profesores del siglo XXI necesitan desarrollar para responder a los retos a los que se están enfrentando en la actualidad. La analítica de datos de aprendizaje pone la tecnología al servicio de la educación. El profesor-investigador obtiene recursos para evaluar su práctica educativa basándose en información estadística: lo que se mide se puede mejorar. El tránsito de la retroalimentación a la autorregulación y la influencia de la EFC en el
Las herramientas digitales no son
la panacea; son solo eso, herramientas. Sin embargo, en manos de docentes creativos, conscientes de la importancia de su labor, permiten generar círculos virtuosos de aprendizaje que impulsan el proceso formativo de construcción del conocimiento binomio enseñanza-aprendizaje son temas que merecen mucha investigación, tanto cuantitativa como cualitativa. Las herramientas digitales no son la panacea; son solo eso, herramientas. Sin embargo, en manos de docentes creativos, conscientes de la importancia de su labor, permiten generar círculos virtuosos de aprendizaje que impulsan el proceso formativo de construcción del conocimiento.

\section{Referencias bibliográficas}

Alcalá, D. H., Pueyo, Á. P. y Calvo, G. G. (2019). Pero... ¿A qué nos referimos realmente con la evaluación formativa y compartida?: confusiones habituales y reflexiones prácticas. Revista Iberoamericana de Evaluación Educativa, 12(1), 13-27.
Cosi, S., Voltas, N., Lázaro-Cantabrana, J. L., Morales, P., Calvo, M., Molina, S. y Quiroga, M. Á. (2020). Formative assessment at university through digital technology tools. Profesorado. Revista de Currículum y Formación del Profesorado, 24(1), 
164-183. https://doi.org/10.30827/profeso rado.v24i1.9314

Díaz, F. y Hernández, G. (2002). Estrategias docentes para un aprendizaje significativo: una interpretación constructivista. Vol. 2. (2. ${ }^{a}$ ed.). McGraw-Hill

Frade, L. (2011). Elaboración de rúbricas: metacognición y aprendizajes. (3. ${ }^{a}$ ed.). Medición de Calidad SA de CV.

García-Jiménez, E. (2015). La evaluación del aprendizaje: de la retroalimentación a la autorregulación. El papel de las tecnologías. RELIEVE. Revista Electrónica de Investigación y Evaluación Educativa, 21(2), 1-24.

Hattie, J. y Timperley, H. (2007). The power of feedback. Review of Educational Research, 77(1), 81-112.
Nicol, D. J. y Macfarlane-Dick, D. (2006). Formative assessment and self-regulating learning: a model and seven principles of good feedback practice. Studies in Higher Education, 31(2), 199-218.

Ravela, P., Picaroni, B. y Loureiro, G. (2017). ¿Cómo mejorar la evaluación en el aula?: reflexiones y propuestas de trabajo para docentes. Instituto Nacional para la Evaluación de la Educación.

Scriven, M. (1967). The methodology of evaluation. Social Science Education Consortium Publication 110.

Wiliam, D. (30 de julio-6 de agosto de 2000). Integrating formative and summative functions of assessment. [Ponencia]. The 9th Educational Congress on Mathematical Education. Tokio, Japón.

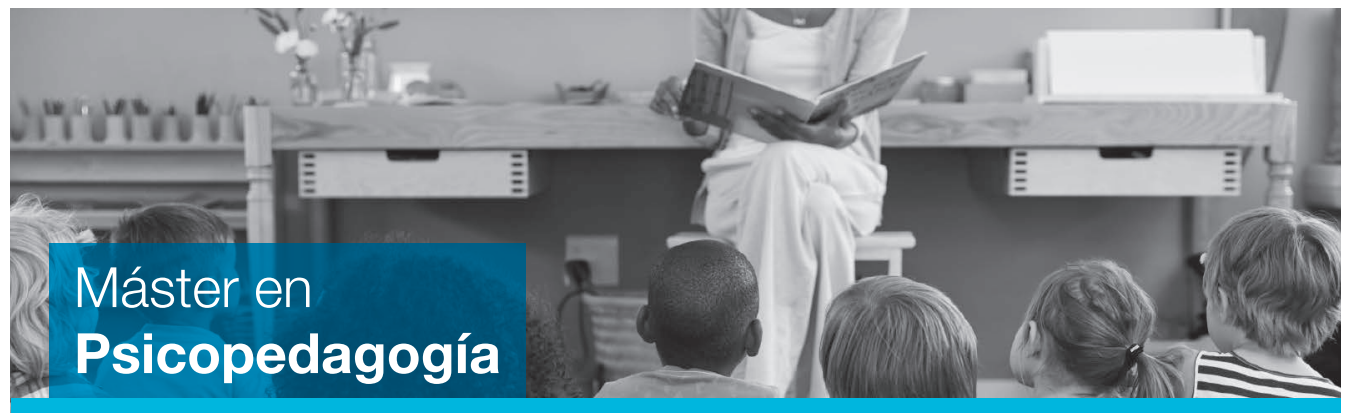

Este máster oficial [60 créditos ECTS] tiene una duración normal de 12 meses.

Dirigido a: Personas vinculadas con el mundo de la educación formal y no formal que deseen actualizar sus conocimientos. El estudiante de este máster ha de estar interesado por la labor del educador en un enfoque educativo inclusivo en el ámbito de la educación formal, y en el trabajo con diferentes grupos o colectivos sociales, favoreciendo la mejora de sus condiciones de vida y la disminución de las desigualdades por motivos de carácter social y cultural.

Objetivos: Permite el desempeño de una labor profesional especializada, avanzada y focalizada en el análisis, la planificación y la intervención para la mejora de los contextos educativos, sociolaborales y sociocomunitarios, de ahí la necesidad de una formación de posgrado que permita el desarrollo de las competencias específicas y multidisciplinares requeridas para su práctica profesional. Pretende dar cobertura a las funciones básicas de los psicopedagogos en distintos ámbitos. 


\section{Instituto de Idiomas}

\section{Metodología personalizada con resultados de aprendizaje garantizados}

La formación de lenguas extranjeras se lleva a cabo dentro de la normativa vigente de la enseñanza de idiomas en España y en la Comunidad Europea. Los cursos presentan un diseño adaptado al Marco Común Europeo de Referencia para las Lenguas y a la metodología propia de la UDIMA: un sistema de enseñanza cercano, flexible, actual, dinámico y personalizado.

\section{Cursos generales}

\section{Cursos universitarios de español}

Títulos propios no oficiales que tratan de acercar al alumno a las habilidades lingüísticas necesarias para el dominio del español como lengua extranjera.

\section{Curso de chino (nivel iniciación)}

Este curso permite que los estudiantes adquieran las capacidades necesarias para defenderse en situaciones sencillas de la vida cotidiana y para desenvolverse socialmente en el idioma chino. Además, prepara al alumno para el examen oficial de primer nivel HSK1/A1.

\section{Cursos de preparación de exámenes}

\section{Certificate in Advanced English (CAE)}

La finalidad del Certificate in Advanced English (CAE) es proporcionar a estudiantes y profesores de idiomas, en una variedad de situaciones, el acceso a una amplia gama de exámenes internacionales de gran calidad, test y diplomas para profesores que les ayuden a lograr sus metas personales y que repercutan favorablemente en su experiencia de aprendizaje y desarrollo profesional. El Certificate in Advanced English (CAE) se corresponde con el nivel C1 del Marco de Referencia Europeo.

\section{Curso de preparación TKT CLIL Module (TKT)}

Este curso, indicado para profesores que están preparando el examen TKT CLIL, les aportará las herramientas y el conocimiento necesarios para superar el examen oficial y para mejorar el proceso de enseñanza-aprendizaje en el aula CLIL. El curso se dará en inglés, ayudando al alumno a reforzar el vocabulario, la gramática y las funciones que han sido aprendidos previamente con explicaciones y correcciones puntuales. Los contenidos se centrarán en los temas necesarios para dar una clase CLIL (principios teóricos, planificación en el aula, técnicas y evaluación).

\section{Preliminary English Test (PET)}

El Preliminary English Test (PET) es un examen de inglés de nivel intermedio. La preparación de este examen ayudará a mejorar las destrezas lingüísticas y el uso del inglés para comunicarse con hablantes nativos de manera cotidiana. El Preliminary English Test (PET) se correponde con el nivel B1 del Marco de Referencia Europeo.

\section{First Certificate in English (FCE)}

La finalidad del First Certificate in English (FCE) es proporcionar a los estudiantes de idiomas la preparación suficiente que les permita lograr sus metas personales, repercutiendo favorablemente en su experiencia de aprendizaje y desarrollo profesional. El First Certificate in English (FCE) se corresponde con el nivel B2 del Marco de Referencia Europeo. 